\title{
Topological, Smooth, and Control Techniques for Perturbed Systems
}

\section{Fritz Colonius and Wolfgang Kliemann}

\begin{abstract}
The theory of dynamical systems has become a center piece in the systematic study of systems with deterministic or stochastic perturbations, based on measurable, topological, and smooth dynamics. Recent developments also forge a close connection between control theory and topological and smooth dynamics. On the other hand, the support theorem of Stroock and Varadhan shows how control theoretic techniques may aid in the Markovian analysis of systems perturbed by diffusion processes. This paper presents an overview of topological, smooth, and control techniques and their interrelations, as they can be used in the study of perturbed systems. We concentrate on global analysis and parameter dependent perturbation systems, where we emphasize comparison of the Markovian and the dynamical structure of systems with Markovian diffusion perturbation process. A series of open problems highlights the areas in which the interconnections between different techniques and system classes are not (yet) well understood.
\end{abstract}

\section{Introduction}

Dynamical systems theory has become a center piece in the study of perturbed systems: Differential equations with deterministic (time varying) perturbations can be understood as skew product flows (see [38]), systems with stochastic perturbations as flows over a probability space (see [8]), and (open loop) control systems as flows over the space of admissible control functions (see [10]). The common feature of these approaches is that perturbed systems are viewed as specific skew product flows, in which the structure of the base flow determines the nature of the perturbation under consideration and the kind of techniques that are appropriate for the analysis of the systems.

At the same time, direct connections between different classes of perturbed systems have been developed, such as support theorems (see [39] for the Markov diffusion case) connecting stochastic systems with control theory, ergodic theory for families of time varying differential equations 
connecting to properties of stochastic systems (see e.g., [35]), or control concepts that describe the recurrence structure of families of differential equations (see [14]). Hence we have a dense net of interdependencies that has propelled the study of perturbed systems in recent years. The monograph by Ludwig Arnold [3] studies these connections, mainly from the point of view of measurable and smooth dynamics, with applications to stochastic bifurcation theory.

This paper collects some ideas from topological and smooth dynamics and from control theory that seem to be useful in the study of perturbed systems. We start from the topological dynamics of skew product flows (in the form of so-called control flows) and point to possible connections with deterministic and stochastic perturbation systems. For the Markov diffusion perturbation model we compare some results obtained via this flow point of view to those obtained via stochastic analysis and the theory of Markov semigroups. In this, as well as in many other parts of our presentation ideas from control theory serve as a unifying technique.

While the basic theory of random dynamical systems has reached a state of maturity, this cannot be said for many of the interdependencies discussed in our paper. The reader will find a variety of open problems and question marks throughout this article for which we would like to know the solutions and answers. We hope that these questions generate further interest in this exciting area.

The analysis of perturbed dynamical systems is concerned mainly with two circles of ideas, namely the global theory and linearization theory, based on spectral concepts. Here we discuss aspects of global theory, such as Morse decompositions, connections between Morse sets, and the behavior of systems on Morse sets. These concepts of topological dynamics are based on chains for (skew product) flows and they are applied to perturbed systems in the first part of Section 3. Regular perturbed systems satisfy a Lie algebra rank condition of the type (14). For these systems the limit sets of the unperturbed flow are enlarged by the perturbation to sets with nonvoid interior in the state space. The global behavior of these systems can be analyzed via the trajectories of associated control systems as explained in the second part of Section 3. One ends up with two global structures for perturbed systems, one based on chains and one on trajectories. Under the so-called inner pair condition (28) we show in Section 4 that these two structures agree 'almost always'. The argument is based on the analysis of parameter dependent systems, which also hints at a bifurcation study of the global behavior of perturbed systems.

A standing assumption throughout this paper is the compactness of the perturbation range and of the state space of the system. We have chosen this set-up, because it implies the existence of limit sets for all system trajectories. This allows us to simplify the formulation of many results and a comparison of the different techniques for various classes of perturbed systems becomes particularly illuminating. We refer the reader to [14] for the 
corresponding results on systems with noncompact state space. If the perturbation range is noncompact (such as the 'white noise' case for stochastic systems), some of the interconnections discussed here remain valid (e.g. those based on the support theorem, see [29]), but the systematic use of topological dynamics would not be possible without restrictions.

This paper presents an overview of topological, smooth and control techniques as they can be used in the study of deterministic and stochastic perturbation systems. We concentrate on the study of global behavior and the connection between topological and control techniques via parameter dependence. An overview over the linearization approach, including spectral concepts for perturbed systems, their associated linear subbundles and invariant manifolds, will appear elsewhere. Most results in this paper are not new. But we hope that our specific presentation of key concepts and their interrelations provides new insights and encourages new research in the area of stochastic dynamics.

\section{Stochastic Systems, Control Flows, and Diffusion Processes: Basic Concepts}

Perturbed systems, as we understand them in this paper, consist of two components, namely the perturbation model and the system model. A natural framework for these systems are skew product flows, which we consider the starting point of our theory. In this section we recall several classes of perturbed systems and describe their relation to skew product flows. An important aspect of our set-up is that all spaces and the dynamical systems on them have topological properties which aid in the qualitative analysis in the subsequent sections.

On the most abstract level, a perturbation model is given by a continuous flow on a topological space $\mathcal{U}$

$$
\theta: \mathbb{R} \times \mathcal{U} \rightarrow \mathcal{U}
$$

i.e. it holds that $\theta_{t} \circ \theta_{s}=\theta_{t+s}$ and $\theta_{0}=$ id. (We will often write $\theta_{t}$ for the map $\theta(t, \cdot)$.) Note that the flow (1) is defined on the two sided time interval $\mathbb{R}$, and hence $\theta_{t}^{-1}=\theta_{-t}$ for all $t \in \mathbb{R}$. The model of a system perturbed by $\theta$ is a continuous skew product flow on the topological product space $\mathcal{U} \times M$

$$
\Phi: \mathbb{R} \times \mathcal{U} \times M \rightarrow \mathcal{U} \times M, \quad \Phi_{t}(u, x)=\left(\theta_{t} u, \varphi(t, x, u)\right)
$$

whose first component, the perturbation (1), affects the system component $\varphi$, but not vice versa. In particular, the $\varphi$-component itself is not a flow. The skew product flow $\Phi$ is a prototype of a deterministically perturbed system in continuous time. In a stochastic perturbation model one has, 
in addition to (2), a probability measure $P$ on the Borel $\sigma$-algebra of $\mathcal{U}$, which is invariant under the flow $\theta$, i.e., $\theta_{t} P=P$ for all $t \in \mathbb{R}$. This set-up differs from the one treated by Arnold [3] in the way that we require $\mathcal{U}$ to be a topological space and $\theta$ to be continuous, while Arnold's perturbation model is just measurable.

The specific perturbations treated in this paper are $L^{\infty}$-functions with compact range. In the deterministic case (1) we consider the following setup:

Let $U \subset \mathbb{R}^{m}$ be compact and convex, with $0 \in \operatorname{int} U$, the interior of $U$. Denote by $\mathcal{U}=\{u: \mathbb{R} \rightarrow U$, measurable $\}$ the perturbation space, equipped with the weak* topology of $L_{\infty}\left(\mathbb{R}, \mathbb{R}^{m}\right)=\left(L_{1}\left(\mathbb{R}, \mathbb{R}^{m}\right)\right)^{*}$. This space is compact and metrizable ([14], Lemma 4.2.1). The flow $\theta$ is given by the standard shift

$$
\theta: \mathbb{R} \times \mathcal{U} \rightarrow \mathcal{U}, \quad \theta_{t}(u(\cdot))=u(t+\cdot),
$$

resulting in a continuous dynamical system ([14, Lemma 4.2.4]). Some standard interpretations of the model (3) are time varying perturbations with a given range, as they are used in robustness theory, or open loop control functions, as they are common in control theory. In a stochastic perturbation model we are also given a $\theta$-invariant probability measure $P$ on $\mathcal{U}$. One way to arrive at such a measure is given by the Kolmogorov construction for stationary processes: Let $\eta: \mathbb{R} \times \Omega \rightarrow U$ be a stationary stochastic process on a probability space $\left(\Omega, \mathcal{F}^{\prime}, P^{\prime}\right)$, with continuous trajectories. Let $\mathcal{C}(\mathbb{R}, U)$ be the space of continuous functions in $\mathbb{R}$ with values in $U$, and $\overline{\mathcal{T}}$ the $\sigma$-algebra on $\mathcal{C}(\mathbb{R}, U)$, generated by the cylinder sets. Then the process $\eta$ induces a probability measure $\bar{P}$ on $(\mathcal{C}(\mathbb{R}, U), \bar{F})$, which is invariant under the shift in $\mathcal{C}(\mathbb{R}, U)$. We imbed $\mathfrak{C}(\mathbb{R}, U)$ into $\mathcal{U}$, extend $\overline{\mathcal{F}}$ to the Borel $\sigma$-algebra $\mathcal{F}$ of $\mathcal{U}$, and extend $\bar{P}$ to a measure $P$ on $\mathcal{F}$, which is invariant under the shift $\theta$ in (3). Compare [22] for details on Kolmogorov's construction. Note that the extension of the trajectory space to $\mathcal{U}$ allows us to use topological properties of the flow $\theta$ in (3).

The specific systems treated in this paper are smooth systems with affine perturbations. Let $M$ be a paracompact $C^{\infty}$-manifold of dimension $d<\infty$, and let $X_{0}, X_{1}, \ldots X_{m}$ be $C^{\infty}$-vector fields on $M$. The system dynamics are given by the ordinary differential equation

$$
\dot{x}=X_{0}(x)+\sum_{i=1}^{m} u_{i}(t) X_{i}(x) \text { on } M .
$$

where $u(\cdot) \in \mathcal{U}$.

Since we restrict ourselves to global flows, we assume that (4) has a unique solution $\varphi(t, x, u)$ for all $(u, x) \in \mathcal{U} \times M$ with $\varphi(0, x, u)=x$, which is defined for all $t \in \mathbb{R}$. Sufficient conditions for this are, e.g., globally Lipschitz continuous vector fields or compactness of $M$, since we assume $U$ 
to be compact. Equation (4) together with the perturbation (3) define the system flow

$$
\Phi: \mathbb{R} \times \mathcal{U} \times M \rightarrow \mathcal{U} \times M, \quad \Phi_{t}(u, x)=\left(\theta_{t} u, \varphi(t, x, u)\right),
$$

which is a continuous skew product flow, compare [14, Lemma 4.3.2]. The topological study of (5) yields some of the basic ideas for the following sections.

The rest of this introductory part is devoted to Markov diffusion systems and to approaches for the analysis of their qualitative behavior. We start from a stochastic perturbation given by a stochastic differential equation on a $C^{\infty}$-manifold $N$ (of finite dimension)

$$
d \eta=Y_{0}(\eta) d t+\sum_{j=1}^{\ell} Y_{j}(\eta) \circ d W_{j}
$$

where $Y_{0}, Y_{1}, \ldots Y_{\ell}$ are $C^{\infty}$-vector fields on $N$ and ' $O$ ' denotes the symmetric (Stratonovich) stochastic differential. (We refer the reader to [2] and [25] for basic facts on stochastic differential equations.) We assume that Equation (6) admits at least one stationary Markov solution, see e.g. [28]. We force this solution to be the unique stationary Markov one by imposing a Lie algebra rank condition of the form

$$
\operatorname{dim} \mathcal{L} \mathcal{A}\left\{Y_{1}, \ldots, Y_{\ell}\right\}(q)=\operatorname{dim} N \text { for all } q \in N
$$

In (7) we have used the following notation: Let $X(N)$ be the set of vector fields on $N$, and let $y \subset X(N)$ be a subset. $\mathcal{L} \mathcal{A}(y)$ denotes the Lie algebra generated by $y$ in $X(N)$, which induces a distribution $\Delta$ (in the differential geometric sense) in the tangent bundle $T N$. For $q \in N$, the vector space $\mathcal{L} \mathcal{A}\{y\}(q) \subset T_{q} N$ is the distribution $\Delta$ evaluated at $q$. Condition (7) guarantees (see [31]) that Equation (6) has a unique stationary Markov solution $\eta_{t}^{*}$ which we extend to all $t \in \mathbb{R}$, compare [3]. We consider this process $\eta_{t}^{*}$ as a background noise, which is mapped via a surjective function

$$
f: N \rightarrow U
$$

onto the perturbation space $U \subset \mathbb{R}^{m}$, compare Lemma 3.17. Then $\xi_{t}=$ $f\left(\eta_{t}^{*}\right)$ is a stationary stochastic process on $U$. Combining this perturbation model with the system (4) we arrive at the Markov diffusion process

$$
\begin{aligned}
d \eta & =Y_{0}(\eta) d t+\sum_{j=1}^{\ell} Y_{i}(\eta) \circ d W_{j}, \quad \eta_{0}=\eta_{0}^{*}, \\
\dot{x} & =X_{0}(x)+\sum_{i=1}^{m} f_{i}\left(\eta_{t}\right) X_{i}(x)
\end{aligned}
$$

on the state space $N \times M$.

The behavior of the system (9) can now be studied using a variety of approaches: 
- Stochastic analysis, compare, e.g., the standard references [25] or [21],

- Stochastic flows, compare [3],

- Imbedding of the stationary process $\eta_{t}^{*}$ into the flow (5) as described above,

- Connections with control theory via the support theorem of Stroock and Varadhan [39].

In this paper we will use a combination of the last two approaches. To this end we briefly describe a version of the support theorem that is suitable for our purposes, compare [30], [32], [24] or [3].

Let $L$ be a finite dimensional $C^{\infty}$-manifold and consider the stochastic differential equation

$$
d z=Z_{0}(z) d t+\sum_{k=1}^{r} Z_{k}(z) \circ d W_{k}
$$

with $C^{\infty}$ vector fields $Z_{0}, \ldots, Z_{r}$. Denote by $\mathcal{C}_{p}\left(\mathbb{R}^{+}, L\right)$ the space of continuous functions $w:[0, \infty) \rightarrow L$ with $w(0)=p \in L$, equipped with the topology of uniform convergence on compact time intervals. For the initial value $p \in L$, the stochastic differential equation induces a probability measure $P_{p}$ on $\mathcal{e}_{p}\left(\mathbb{R}^{+}, L\right)$ which, intuitively, assigns to each Borel set $B$ in $\mathrm{e}_{p}\left(\mathbb{R}^{+}, L\right)$ the probability that the functions in $B$ appear as trajectories of the solution of (10). Stroock and Varadhan [40] associate with (10) formally a control system of the form

$$
\dot{z}=Z_{0}(z)+\sum_{k=1}^{r} w_{k}(t) Z_{k}
$$

with control functions $w \in \mathcal{W}=\left\{w:[0, \infty) \rightarrow \mathbb{R}^{r}\right.$, piecewise constant $\}$. We denote by $\psi(\cdot, p, w)$ the solutions of (11) with initial value $\psi(0, p, w)=p$, and by $\Psi_{p}=\{\psi(\cdot, p, w), w \in \mathcal{W}\} \subset \mathcal{e}_{p}\left(\mathbb{R}^{+}, L\right)$ the set of all such solutions. The support theorem now states

$$
\operatorname{supp} P_{p}=\mathrm{cl} \Psi_{p}
$$

where 'supp' denotes the support of a measure (i.e. the smallest closed subset of full measure), and the closure 'cl' is taken in $\mathcal{C}_{p}\left(\mathbb{R}^{+}, L\right)$. In the form (12) the support theorem is not yet suitable for the study of (9) with $L=N \times M$, because it refers only to fixed initial conditions, the control functions in $\mathcal{W}$ are taken to be piecewise constant, and we would have to choose controls with values in $\mathbb{R}^{\ell}$ to first analyze the $\eta$, and then the $x-$ component of (9). However, Kunita [31] shows that under the Lie algebra rank condition $\operatorname{dim} \mathcal{L} \mathcal{A}\left\{Z_{1}, \ldots, Z_{r}\right\}(p)=\operatorname{dim} L$ for all $p \in L$ we have

$$
\operatorname{cl} \Psi_{p}=\bigodot_{p}\left(\mathbb{R}^{+}, L\right) .
$$


This, together with an appropriate concept of controllability regions, will allow us to reduce the control analysis to the system (4) with control functions in $\mathcal{U}$, and hence to the study of the skew product flow $(5)$.

\section{Attractors, Invariant Measures, Control, and Chaos}

Global analysis of dynamical systems deals with limit sets, connections between limit sets, and the behavior of the system on limit sets. This information is pieced together to obtain a global picture of the system behavior for $t \rightarrow \infty$ and $t \rightarrow-\infty$. As it turns out, limit sets can be rather complicated, particularly for systems with time varying perturbations, such as (4) and (9). Therefore it is useful to study more robust concepts, such as versions of recurrence or attractors that do allow a global analysis for larger classes of systems. In our context of perturbed systems it will turn out that some control theoretic concepts simplify the study of recurrence and of attractors, and that one obtains a fairly complete picture for Markov diffusion models. We develop this theory stepwise, starting with general recurrence concepts, the study of the perturbation model (3), and of the system model (5), before we proceed to stochastic systems.

\subsection{Concepts from Topological Dynamics}

Throughout this section we avoid questions about the existence of limit sets by assuming that the state space $M$ of the system is compact. Generalizations of the basic results to the noncompact case can be found, e.g. in [14]. Let $S$ be a compact metric space and let $\Psi: \mathbb{R} \times S \rightarrow S$ be a continuous flow. For $V \subset S$ we denote the $\omega$-limit set by $\omega(V)=\{x \in S$, there are $x_{k} \in V$ and $t_{k} \rightarrow \infty$ with $\left.\Psi\left(t_{k}, x_{k}\right) \rightarrow x\right\}$, and similarly for the $\alpha$-limit set $\omega^{*}(V)$, using $t_{k} \rightarrow-\infty$.

Definition 3.1. The flow $\Psi$ is called topologically transitive if there exists $x \in S$ with $\omega(x)=S$, and topologically mixing if for any two open sets $V_{1}, V_{2} \subset S$ there exists $t>0$ with $\Psi\left(-t, V_{1}\right) \cap V_{2} \neq \emptyset$.

Note that a topologically mixing flow is topologically transitive. These topological concepts are based on the trajectories of the flow and on limit sets. One obtains more robust concepts by considering chains instead of trajectories.

Definition 3.2. For $x, y \in S$ and $\varepsilon, T>0$ an $(\varepsilon, T)$-chain from $x$ to $y$ is given by a number $n \in \mathbb{N}$, points $x_{0}=x, x_{1}, \ldots, x_{n}=y$ in $S$ and times $t_{0}, \ldots, t_{n-1} \geq T$ such that $d\left(\Psi\left(t_{i}, x_{i}\right), x_{i+1}\right)<\varepsilon$ for $i=0, \ldots, n-1$. 
For $V \subset S$ we denote the chain limit set by $\Omega(V)=\{x \in S$, for all $\varepsilon, T>$ 0 there exists $y \in V$ and an $(\varepsilon, T)$ - chain from $y$ to $x\}$. Using chains one can formulate the following recurrence concepts.

Definition 3.3. A subset $V \subset S$ is chain transitive, if for all $x, y \in V$ we have $x \in \Omega(y)$. A point $x \in S$ is chain recurrent if $x \in \Omega(x)$. We denote the set of all chain recurrent points by $\mathcal{R}$.

One can show that a closed subset $V \subset S$ is chain transitive iff it is chain recurrent and connected. Furthermore, the connected components of the chain recurrent set $\mathcal{R}$ coincide with the maximal chain transitive subsets of $\mathcal{R}$.

Finally, we introduce Morse decompositions of the flow $(S, \Psi)$.

Definition 3.4. A Morse decomposition of $(S, \Psi)$ is a finite collection $\left\{\mathcal{M}_{i}, i=1 \ldots n\right\}$ of nonvoid, pairwise disjoint compact invariant sets such that

(i) For all $x \in S$ we have $\omega(x), \omega^{*}(x) \subset \bigcup_{i=1}^{n} \mathcal{M}_{i}$,

(ii) Suppose there are $\mathcal{M}_{j_{0}}, \ldots, \mathcal{M}_{j_{\ell}}$ and $x_{1}, \ldots, x_{\ell} \in S \backslash \bigcup_{i=1}^{n} \mathcal{M}_{i}$ with $\omega^{*}\left(x_{i}\right) \subset \mathcal{M}_{j_{i-1}}$ and $\omega\left(x_{i}\right) \subset \mathcal{M}_{j_{i}}$ for $i=1, \ldots, \ell$, then $\mathcal{M}_{j_{0}} \neq \mathcal{M}_{j_{\ell}}$.

The sets of a Morse decomposition are called Morse sets. A Morse decomposition induces an order on the Morse sets through the relation $\mathcal{M}_{i} \preceq \mathcal{M}_{j}$ if there exists $x \in S$ with $\omega^{*}(x) \subset \mathcal{M}_{i}$ and $\omega(x) \subset \mathcal{M}_{j}$.

Morse decompositions describe the flow $\Psi$ via its movement from Morse sets that are smaller (w.r.t. the order $\preceq$ ) to ones that are greater. This gives a fairly complete global picture of the flow $\Psi$ if it has a finest Morse decomposition. The following result clarifies the relation between Morse decompositions and chain recurrence.

Proposition 3.5. The flow $(S, \psi)$ admits a finest Morse decomposition iff the chain recurrent set $\mathcal{R}$ consists of finitely many connected components. In this case, the Morse sets coincide with the (chain recurrent) components of $\mathcal{R}$ and the flow restricted to each Morse set is chain transitive and chain recurrent.

For the proof of this result and for further discussions of the concepts above see, e.g. [1], [27], [36], or Appendix B in [14].

\subsection{Deterministic Perturbed Systems}

In the next step we apply the recurrence concepts $3.1-3.4$ to the perturbation model (3), compare [14], Proposition 4.2.7.

Proposition 3.6. Consider the shift system $\theta: \mathbb{R} \times \mathcal{U} \rightarrow \mathcal{U}$ as defined in (3). This flow is topologically mixing, topologically transitive, and chain 
transitive. In particular, $(\mathcal{U}, \theta)$ admits only the trivial Morse decomposition $\{\mathcal{U}\}$.

As a corollary we obtain that the shift $(\mathcal{U}, \theta)$ is also topologically chaotic in the sense of Devaney [19].

Definition 3.7. A continuous flow $(S, \Psi)$ is called topologically chaotic, if

(i) $\Psi$ is topologically mixing,

(ii) $\Psi$ has a dense set of periodic points,

(iii) $\Psi$ has sensitive dependence on initial conditions, i.e. there exists $\delta>$ 0 such that for all $x \in S$ and neighborhoods $N$ of $x$ there are $y \in N$ and $t>0$ such that $d(\Psi(t, x), \Psi(t, y))>\delta$.

Corollary 3.8. The shift system $\theta: \mathbb{R} \times \mathcal{U} \rightarrow \mathcal{U}$ from (3) is topologically chaotic, if $U$ consists of more than one point.

The proof of Corollary 3.8 uses the fact that the periodic functions are dense in $\mathcal{U}$ (see [14, Lemma 4.2.2], which together with topological transitivity implies sensitive dependence on initial conditions ([14, Prop. B.2.6]).

Our next step is the study of the system flow $\Phi: \mathbb{R} \times \mathcal{U} \times M \rightarrow \mathcal{U} \times M$ as defined in (5). The global behavior of this flow can be much more intricate than the one of the perturbation alone. It turns out that control theoretic concepts help in the analysis of $\Phi$ and we introduce the basic ideas next.

Consider the system dynamics (4) as a control system with state space $M$ and admissible control functions $u \in \mathcal{U}$. We impose a nondegeneracy condition on (4) which implies that $M$ is the 'right' state space:

$$
\operatorname{dim} \mathcal{L} \mathcal{A}\left\{X_{0}+\Sigma u_{i} X_{i}, u \in U\right\}(x)=\operatorname{dim} M \text { for all } x \in M
$$

Condition (14) implies that the positive (and negative) orbits from each point have nonvoid interior, i.e. int $\mathcal{O}^{+}(x) \neq \emptyset$ for all $x \in M$, where $\mathcal{O}^{+}(x)=$ $\{y \in M$, there exist $t \geq 0$ and $u \in \mathcal{U}$ with $y=\varphi(t, x, u)\}$, and similarly for the negative orbit $\mathcal{O}^{-}(x)$ using times $t \leq 0$. We remark that the orbits are finite time objects of a control system ('steering $x$ to $y$ in time $t$ with an appropriate control'), but for any $u \in \mathcal{U}$ it also holds that the limit set $\omega(u, x)$ of the trajectory $\varphi(\cdot, x, u)$ in $M$ is contained in $\mathrm{cl}^{+}(x)$, the closure of the positive orbit. Furthermore, we have the following result, which is an easy consequence of the continuous dependence of the solution of a differential equation on the right hand side.

Lemma 3.9. For each point $x \in M$ the closure $\mathrm{cl}^{+}(x)$ of its forward orbit agrees with the closure of the forward orbit defined via piecewise continuous, piecewise constant, continuous, or $C^{\infty}$ controls. The same holds for $\mathrm{cl}^{-}(x)$. 
For further information on control theoretic ideas see, e.g., [26] or Appendix $\mathrm{A}$ in [14]. We now define the basic objects that are useful for the global analysis of perturbed systems.

Definition 3.10. A set $D \subset M$ is called a control set of the system (4) if

(i) for all $x \in D$ there exists $u \in \mathcal{U}$ with $\varphi(t, x, u) \in D$ for all $t \geq 0$,

(ii) for all $x \in D$ one has $D \subset \operatorname{cl} \mathcal{O}^{+}(x)$,

(iii) D is maximal (w.r.t. set inclusion) with the properties (i) and (ii).

$A$ control set $C$ is called invariant if $\mathrm{cl} C=\mathrm{cl}^{+}(x)$ for all $x \in C$, and $D \subset M$ is a main control set if it is a control set with int $D \neq \emptyset$.

Note that according to Lemma 3.9 control sets are independent of the class of control functions listed in the lemma.

In order to obtain a complete picture of the global behavior of control systems, we introduce two concepts related to control sets:

The domain of attraction of a control set $D$ is defined as

$$
\mathbf{A}(D)=\left\{y \in M, c l 0^{+}(y) \cap D \neq \emptyset\right\}
$$

The reachability order on the control sets of (4) is given by

$$
D \preceq D^{\prime} \text { if } D \cap \mathbf{A}\left(D^{\prime}\right) \neq \emptyset
$$

The following result characterizes the global behavior of control systems on compact spaces, compare [14, Chapter 3] for the noncompact case.

Theorem 3.11. Consider the control system (4) on the compact space $M$ and assume the Lie algebra rank condition (14).

(i) There exist at least one closed main control set $C$ and one open main control set $C^{*}$.

(ii) A main control set is closed iff it is invariant. The closed main control sets are exactly the maximal sets under the order $\preceq$.

(iii) The open control sets are exactly the minimal sets under the order $\preceq$.

(iv) There are finitely many closed and finitely many open main control sets.

This theorem is an easy consequence of [14, Th. 3.15]. Theorem 3.11 describes the 'flow' of a control system from the minimal, open control sets to the maximal, closed ones along the order $\preceq$. Hence if suffices to know the control sets and their order to obtain the picture of the global behavior w.r.t. the orbits of a control system. The study of the global behavior of the individual trajectories requires some knowledge about the system flow (5), compare Proposition 3.22 below. 
The closures of the positive and negative orbits contain all limit sets of trajectories in $M$ (see the remark after (14)). However, limit sets are not necessarily contained in the closures of main control sets. Therefore, we need an analogue of control sets, but defined via chains.

Definition 3.12. Fix $x, y \in M$ and pick $\varepsilon, T>0$. A controlled $(\varepsilon, T)-$ chain $\zeta$ from $x$ to $y$ is given by $n \in \mathbb{N}, x_{0}=x, x_{1}, \ldots, x_{n}=y$ in $M$, $u_{0}, \ldots, u_{n-1}$ in $\mathcal{U}$ and $t_{0}, \ldots, t_{n-1} \geq T$ such that $d\left(\varphi\left(t_{j}, x_{j}, u_{j}\right), x_{j+1}\right)<\varepsilon$ for all $j=0, \ldots, n-1$.

Definition 3.13. A set $E \subset M$ is called a chain control set of (4) if

(i) for all $x \in E$ there exists $u \in \mathcal{U}$ with $\varphi(t, x, u) \in E$ for all $t \in \mathbb{R}$,

(ii) for all $x, y \in E$ and $\varepsilon, T>0$ there is a controlled $(\varepsilon, T)$-chain from $x$ to $y$,

(iii) E is maximal (w.r.t. set inclusion) with the properties (i) and (ii).

For basic properties of chain control sets see [14, Section 3.4].

With these control theoretic preparations we are ready to study the flow (5) of a perturbed system. We lift the main control sets $D$ and the chain control sets $E$ from the state space $M$ to the product space $\mathcal{U} \times M$ :

$$
\begin{aligned}
\mathcal{D} & =c l\{(u, x) \in \mathcal{U} \times M, \varphi(t, x, u) \in \operatorname{int} D \text { for all } t \in \mathbb{R}\} \\
\mathcal{E} & =\{(u, x) \in \mathcal{U} \times M, \varphi(t, x, u) \in E \text { for all } t \in \mathbb{R}\}
\end{aligned}
$$

Theorem 3.14. Consider the system flow (5) and assume the Lie algebra rank condition (14). Let $\mathcal{D} \subset \mathcal{U} \times M$ be compact such that the projection $\pi_{M} \mathcal{D}=\{x \in M$, there exists $u \in \mathcal{U}$ with $(u, x) \in \mathcal{D}\}$ has nonvoid interior.

(i) $\mathcal{D}$ is maximal topologically mixing iff there is a main control set $D \subset$ $M$ whose lift in the form (17) agrees with $\mathcal{D}$.

(ii) Statement (i) remains true for $\mathcal{D}$ maximal topologically transitive.

(iii) If $U$ contains more than one point, then for any lift $\mathcal{D}$ of a main control set the flow $\left(\mathcal{D}, \Psi_{\mid \mathcal{D}}\right)$ is topologically chaotic.

For the proof of this theorem see [14, Prop. 4.3.3, Th. 4.3.8, Cor. 4.3.9]. Theorem 3.14 establishes for perturbed systems the correspondence of controllability, topological transitivity, and topological chaos. The reachability order between control sets induces an order between the topologically transitive components of the perturbation flow. A similar relationship holds for the chain transitive components.

Theorem 3.15. Consider the system flow (5). Then $\mathcal{E} \subset \mathcal{U} \times M$ is a maximal invariant chain transitive set iff $\pi_{M} \mathcal{E}$ is a chain control set. In this case the lift of $\pi_{M} \mathcal{E}$ is equal to $\mathcal{E}$. 
A proof of this result can be found in [14, proof of Th. 4.3.11]. Combining Theorem 3.15 with Proposition 3.5 we obtain the following consequence.

Corollary 3.16. Assume that the control system (4) has finitely many chain control sets $E_{1}, \ldots, E_{k}$. Then the lifts $\left\{\mathcal{E}_{1}, \ldots, \mathcal{E}_{k}\right\}$ are the (unique) finest Morse decomposition of the system flow $(\mathcal{U} \times M, \Phi)$. Furthermore, the order on this Morse decomposition (compare Definition 3.4) induces an order between the chain control sets of (4).

The results $3.14-3.16$ show two pictures of the global topological behavior of the system flow (5), one with respect to controllability and topologically chaotic components, the other one with respect to chain recurrence and Morse decompositions. Under an additional assumption we will merge these two pictures into one in the next section.

\subsection{Global Behavior of Markov Diffusion Systems}

The rest of this section is devoted to consequences of the theory above for stochastically perturbed systems, including a discussion of invariant measures and attractors. Obviously, the global behavior of a stochastic system, i.e. a system with an additional $\theta$-invariant probability measure $P$ on the perturbation flow (3), has to follow the lines of the topological results above. However, depending on the support and the specific form of the measure $P$ a multitude of specific patterns is possible. Therefore we begin this discussion with the Markov diffusion model (9), which shows a particularly simple behavior, completely described by the control theoretic results on the system (4).

We need two preparatory results. Consider the control system associated with (9) in the sense of Stroock and Varadhan (compare (10), (11)), i.e.

$$
\begin{aligned}
& \dot{\eta}=Y_{0}(\eta)+\sum_{j=1}^{\ell} w_{j}(t) Y_{j}(\eta) \quad \text { on } N \\
& \dot{x}=X_{0}(x)+\sum_{i=1}^{m} f_{i}(\eta(t)) X_{i}(x)=: X(x, \eta) \text { on } M
\end{aligned}
$$

where $f: N \rightarrow U$ is of the form (8), w $\quad$ W $=\left\{w:[0, \infty) \rightarrow \mathbb{R}^{\ell}\right.$, piecewise constant\}, and assume the Lie algebra rank condition (7) for the $\eta$-component. Furthermore, we assume the weaker Lie algebra rank condition for the pair system

$$
\begin{aligned}
\operatorname{dim} \mathcal{L} A\left\{\left\{\begin{array}{c}
Y_{0}+\Sigma w_{j} Y_{j} \\
X(x, \eta)
\end{array}\right), w \in \mathbb{R}^{\ell}\right\}\left(\begin{array}{l}
\eta \\
x
\end{array}\right)= & \operatorname{dim} N+\operatorname{dim} M \\
& \text { for all }\left(\begin{array}{l}
\eta \\
x
\end{array}\right) \in N \times M
\end{aligned}
$$

This condition implies, in particular, that (14) holds for the $x$-component.

Lemma 3.17. Let $f: N \rightarrow U$ be a continuous map such that there exists a closed, connected subset $L \subset N$ with $\left.f\right|_{L}$ is $C^{1}$ and $D f(\eta)$ has full rank for all $\eta \in L$ with $f(\eta) \in \operatorname{int} U$. Then for all $(\eta, x) \in N \times M$ the orbits 
$\mathcal{O}^{+}(\eta, x)$ of the system (19) are of the form $\mathrm{cl}^{+}(\eta, x)=N \times \mathrm{cl}^{+}(x)$, where $\mathcal{O}^{+}(x)$ is the positive orbit of the system (4) from $x \in M$. In particular, the invariant control sets $\hat{C}$ of (19) correspond one-to-one to the invariant control sets $C$ of (4) via $\hat{C}=N \times C$.

Proof. We start with the following two observations: By Kunita's Theorem (compare (13)), the Lie algebra rank condition (7) for the $\eta$-system implies, that for all $\eta \in N$ every continuous function in $\mathcal{C}_{\eta}\left(\mathbb{R}^{+}, N\right)$ can be approximated by trajectories starting in $\eta$ with controls $w \in \mathcal{W}$. Furthermore, by boundedness of $f$, the $x$-components of the trajectories of the system (19) satisfy $\varphi(t, \eta, x, w) \rightarrow x$ for $t \rightarrow 0$, uniformly for $w \in \mathcal{W}$.

In order to show that for $x \in M$ and $\eta \in N$ one has $N \times \operatorname{cl}^{+}(x) \subset$ $\operatorname{cl} \mathcal{O}^{+}(\eta, x)$, consider $\left(\eta_{1}, x_{1}\right) \in N \times \mathcal{O}^{+}(x)$ with $x_{1}=\varphi(T, x, u) \in \mathcal{O}^{+}(x)$, where $T>0$. We may assume that $u(t) \in \operatorname{int} U$ for all $t \in[0, T]$ and that $u \in \mathcal{U}$ is a continuous control (compare Lemma 3.9). By the Implicit Function Theorem one finds $t_{0}:=0<t_{1}<\ldots<t_{n}=T$, open sets $V_{i} \subset U$, and $C^{1}$ maps $h_{i}: V_{i} \rightarrow N$ such that $f \circ h_{i}=i d$ on $V_{i}$ and $\left[t_{i}, t_{i+1}\right] \subset$ $\left\{t \in[0, T] ; u(t) \in V_{i}\right\}$ for all $i$. Thus $h_{i}(u(t)), t \in\left[t_{i}, t_{i+1}\right]$, is continuous. Clearly, for all $k \in \mathbb{N}$ there is a continuous function on $\left[0, \frac{1}{k}\right]$ connecting $\eta$ and $h_{0}\left(u\left(\frac{1}{k}\right)\right)$; furthermore, there are continuous functions on $\left[t_{i+1}-\frac{1}{k}, t_{i+1}\right]$ connecting $h_{i}\left(u\left(t_{i}-\frac{1}{k}\right)\right)$ and $h_{i+1}\left(u\left(t_{i+1}\right)\right)$; and, finally, there are continuous functions on $\left[t_{n}-\frac{1}{k}, t_{n}\right]$ connecting $h_{n-1}\left(u\left(t_{n}-\frac{1}{k}\right)\right)$ and $\eta_{1}$. Together, we have constructed continuous functions on $[0, T]$ starting in $\eta$ and ending in $\eta_{1}$. The two introductory observations imply that this construction yields trajectories of the coupled system (19) establishing $\left(\eta_{1}, x_{1}\right) \in \operatorname{cl} \mathcal{O}^{+}(\eta, x)$. The converse inclusion is obvious. Now the final assertion on the invariant control sets is a direct consequence of their definition.

If $\operatorname{dim} N=1$, it suffices to assume that the restriction of $f$ to a connected subset of $N$ is continuous and bijective onto $U$.

According to Lemma 3.17 the global control structure of the $x$-component (4) determines the control structure of the pair system (19). As we will see, it is sufficient for the global analysis of the Markov diffusion model to understand the invariant control sets of (4) and the corresponding multistability regions.

Definition 3.18. A point $x \in M$ is called multistable for the system (4) if there exist invariant control sets $C_{1}, C_{2} \subset M$ such that $x \in \mathbf{A}\left(C_{i}\right)$ for $i=$ 1,2. (Compare (15) for the definition of the domain of attraction $\mathbf{A}(C)$.) The set of all multistable points will be denoted by MS.

The set of multistable points is nonempty iff the system (4) has at least two invariant control sets. Furthermore, there exist finitely many control sets $D_{1}, \ldots, D_{k}$ such that $M S=\bigcup_{i=1}^{k} \mathbf{A}\left(D_{i}\right)$. For further information on multistable points and on the characterization of the sets $D_{1}, \ldots, D_{k}$ see $[14$, Section 3.3]. 
The next result characterizes the global behavior of the Markov diffusion model (9). We work on the canonical probability space $\hat{\Omega}=\mathcal{C}\left(\mathbb{R}^{+}, N \times M\right)$ with the induced measures $\hat{P}_{(q, x)}$ for fixed initial conditions $(q, x) \in N \times M$. By $\hat{P}_{\left(\eta^{*}, x\right)}$ we denote the measure corresponding to the stationary Markov solution $\left\{\eta_{t}^{*}, t \geq 0\right\}$ in the $\eta$-component. Its marginal distribution on $\Omega=$ $\mathcal{C}\left(\mathbb{R}^{+}, M\right)$ will be denoted by $P_{x}, x \in M$. The trajectories of the pair process are $(\eta(t, q, \omega), \varphi(t,(q, x), \omega))$ for $(q, x) \in N \times M$, and the $x$-component under the stationary solution $\left\{\eta_{t}^{*}, t \geq 0\right\}$ will be written as $\varphi(t, x, \omega), x \in$ $M$. Finally, for a set $A \subset M$ we introduce the first entrance time of the $x$-component from $x \in M$ as

$$
\tau_{x}(A)=\inf \{t \geq 0, \varphi(t, x, \omega) \in A\} .
$$

With these notations we obtain the following characterization.

Theorem 3.19. Consider the Markov perturbation model (9) under the Lie algebra rank conditions (7) and (20).

(i) The control system (4) has finitely many invariant control sets $C_{1}$, $\ldots, C_{k}$.

(ii) For each $x \in M$ there exist numbers $p_{i}(x) \geq 0, i=1 \ldots k$ with $\sum_{i=1}^{k} p_{i}(x)=1$ and $p_{i}(x)=P_{x}\left\{\tau_{x}\left(C_{i}\right)<\infty\right\}$.

(iii) We have $p_{i}(x)>0$ iff $x \in \mathbf{A}\left(C_{i}\right)$, the domain of attraction of $C_{i}$ (compare (15)), and $p_{i}(x)=1$ iff $x \in \mathbf{A}\left(C_{i}\right) \backslash M S$.

(iv) Each invariant control set is invariant for the process $\{\varphi(t, x, \omega), t \geq$ $0\}$, i.e. $P_{x}\left\{\varphi(t, x, \omega) \in C_{i}\right.$ for all $\left.t \geq 0\right\}=1$ for $x \in C_{i}, i=1 \ldots k$.

(v) Set $C:=\bigcup_{i=1}^{k} C_{i}$, then $\tau_{x}(C)$ has finite expectation for $x \in M$.

Proof. Part (i) is [14, Theorem 3.2.8]. By Lemma 3.17 the invariant control sets (19) are of the form $N \times C_{i}, i=1 \ldots k$. Hence we have $\tau_{x}\left(C_{i}\right)=$ $\inf \left\{t \geq 0,\left(\eta_{t}^{*}, \varphi(t, x, \omega)\right) \in N \times C_{i}\right\}=: \hat{\tau}_{x}\left(N \times C_{i}\right)$ for all $x \in M$, and $P_{x}\left\{\tau_{x}\left(C_{i}\right)<\infty\right\}=\hat{P}_{\left(\eta^{*}, x\right)}\left\{\hat{\tau}_{x}\left(N \times C_{i}\right)<\infty\right\}$. Denote $C=\bigcup_{i=1}^{k} C_{i}$ and observe that $N \times C$ is a disjoint union of $\left(\eta^{*}(t), \varphi(t)\right)$-invariant sets, see (iv). Therefore $\hat{P}_{\left(\eta^{*}, x\right)}\left\{\hat{\tau}_{x}(N \times C)<\infty\right\}=\sum_{i=1}^{k} \hat{P}_{\left(\eta^{*}, x\right)}\left\{\hat{\tau}_{x}\left(N \times C_{i}\right)<\infty\right\}$. But for all $(q, x) \in N \times M$ we have $\hat{P}_{(q, x)}\left\{\hat{\tau}_{x}(N \times C)<\infty\right\}=1$ (see [29]), hence $\left.\hat{P}_{\left(\eta^{*}, x\right)}(N \times C)<\infty\right\}=1$, which proves (ii). Part (iii) follows immediately from the support theorem (13), Proposition 3.22 below and the fact that the distribution of $\eta_{0}^{*}$ has a $C^{\infty}$-density with support equal to $N$. To show (iv) it suffices to prove that for all $i=1 \ldots k$ the sets $N \times C_{i}$ are $\left(\eta_{t}^{*}, \varphi(t)\right)-$ invariant. Since by Lemma 3.17 the sets $N \times C_{i}$ are the invariant control sets of $(19)$, they are $(\eta(t, q, \omega), \varphi(t,(q, x), \omega))$-invariant for all $(q, x) \in$ $N \times C_{i}$, compare [29]. Hence they are $\left(\eta_{t}^{*}, \varphi(t, x, \omega)\right)$-invariant for all $x \in C_{i}$. Finally, (v) is a standard argument using [20, Lemma 4.3], compare [29]. 
Theorem 3.19 characterizes the global behavior of the Markov diffusion model (9): The system enters from any initial value $x \in M$ the invariant control sets of (4) in finite time and and stays there for the rest of its life. The invariant set $C=\bigcup_{i=1}^{k} C_{i}$ is completely determined by control analysis, as is the question of $p_{i}(x)=0$ or $=1$, i.e. whether the system reaches the set $C_{i}$ from $x \in M$ and whether this happens with probability 1. Therefore, these facts are independent of the specific background noise $\eta_{t}^{*}$ and of the map $f$, as long as the Lie algebra rank conditions are satisfied and $f$ is surjective. Of course, if $p_{i}(x) \in(0,1)$, then this quantity does depend on $\eta_{t}^{*}$ and on $f$. Hence one can consider the invariant control sets as 'limit sets' for the Markov diffusion model.

\subsection{Invariant Measures}

It remains to investigate the behavior of the system on the limit sets, i.e. invariant measures and ergodicity. In the Markovian context we are interested in invariant Markov measures: Denote by $\hat{P}_{t}$ the Markovian semigroup of (9) on $\mathcal{C}\left(\mathbb{R}^{+}, N \times M\right)$, a probability measure $\mu$ on $N \times M$ (with the Borel $\sigma$-algebra) is called an invariant Markov measure of (9) if

$$
\hat{P}_{t} \mu=\mu \text { for all } t \geq 0
$$

Theorem 3.20. Consider the Markov perturbation model (9) under the Lie algebra rank conditions (7) and (20).

(i) There exists a unique invariant Markov measure $\nu$ on $N$ for the $\eta-$ component of (9) with supp $\nu=N$.

(ii) For each invariant control set $C_{i}, i=1 \ldots k$ of the system (4) there exists a unique invariant Markov measure $\mu_{i}$ for the pair process (9) with supp $\mu_{i}=N \times C_{i}$. Furthermore, the marginal of $\mu_{i}$ on $N$ is the given measure $\nu$.

(iii) The law of large numbers holds for all measures $\mu_{i}$, i.e. one has for all $g \in L_{1}\left(\mu_{i}\right)$ and for $\mu_{i}$-almost all $(q, x) \in N \times M$

$$
\hat{P}_{(q, x)}\left\{\lim _{T \rightarrow \infty} \frac{1}{T} \int_{0}^{T} g(\eta(t), \varphi(t)) d t=\int_{N \times C_{i}} g(p, y) \mu_{i}(d(p, J))\right\}=1 .
$$

(iv) Under the stationary solution $\left\{\eta_{t}^{*}, t \geq 0\right\}$ in the $\eta$-component we have for all $x \in M:\left(\eta_{t}^{*}, \varphi(t, x, \omega)\right) \Rightarrow \sum_{i=1}^{k} p_{i}(x) \mu_{i}$ as $t \rightarrow \infty$, where $\Rightarrow$ denotes convergence in distribution.

(v) For each $i=1 \ldots k$ the $x$-component has a unique stationary solution $x_{i}^{*}(t)$ on $C_{i}$, which is stationarily connected with $\eta_{t}^{*}$, i.e. $\left(\eta_{t}^{*}, x_{i}^{*}(t)\right)$ is a stationary Markov solution of ( 9 ). 
Furthermore, all invariant measures admit $C^{\infty}$-densities.

Proof. The results (i) - (iii) and the existence of $C^{\infty}$-densities for the invariant measures were proved in [29]. To show (iv) we note first of all that the law of large numbers also holds for $\hat{P}_{\left(\eta^{*}, x\right)}=\int_{N} \hat{P}_{(q, x)} d \nu$. Now the claim follows via standard arguments, see e.g. [29], from Theorem 3.19, if the $\mu_{i}$, $i=1 \ldots k$ are the only invariant Markov measures of $(9)$. But invariant Markov measures of (9) have support on the invariant control sets of (19) and these are exactly the sets of the form $N \times C_{i}$ according to Lemma 3.17. Now the uniqueness part of (ii) shows that $\mu_{i}, i=1 \ldots k$ are the only invariant Markov measures of (9). Part (v) follows from the fact that $\eta_{t}^{*}$-stationary solutions of the $x$-component are in one-to-one correspondence to the invariant Markov measures of the pair process $\left(\eta_{t}, x_{t}\right)$, since components of stationary processes are stationary.

As the results above show, Markov diffusion theory is basically a state space theory in the sense that the qualitative behavior of the Markov perturbation model (9) (stationarity, ergodicity, convergence of the distributions) can be described using state space concepts in $M$ and in $N \times M$. The properties of the associated system flow (5) (compare the remarks after (9)) did not enter into our discussion. However, Theorems 3.19 and 3.20 have some immediate consequences for the limit behavior of the trajectories.

Corollary 3.21. Under the assumptions of Theorem 3.19 and 3.20 we have

(i) For all $x \in M$ there exists a $P_{x}-a$.s. finite random variable $\tau_{x}$ (with finite expectation) such that $P_{x}\left\{\varphi(t, x, \omega) \in C=\bigcup_{i=1}^{k} C_{i}\right.$ for all $t \geq$ $\left.\tau_{x}\right\}=1$.

(ii) For $\mu_{i}$-almost all $(q, x) \in N \times C_{i}$ we have $\hat{P}_{q, x}\left\{\pi_{M} \hat{\omega}(q, x)=C_{i}\right\}=$ 1 , where $\hat{\omega}(q, x)$ denotes the $\omega$-limit set of the trajectory $(\eta(t, q, \omega)$, $\varphi(t,(q, x) \omega)), t \geq 0$.

These almost sure statements for the Markov diffusion model have as counterparts topologically generic statements for the system flow (5):

Proposition 3.22. Consider the system flow under the Lie algebra rank condition (14).

(i) The set $\{(u, x) \in \mathcal{U} \times M$, there exists $T>0$ such that for all $t \geq T$ $\left.\varphi(t, x, u) \in C=\bigcup_{i=1}^{k} C_{i}\right\}$ is open and dense in $\mathcal{U} \times M$.

(ii) For each $i=1 \ldots k$ the set $\left\{(u, x) \in \mathcal{U} \times C_{i}, \pi_{M} \omega(u, x)=C_{i}\right\}$ is residual in $\mathcal{U} \times C_{i}$, i.e. it contains a countable intersection of open and dense subsets of $\mathcal{U} \times C_{i}$. 
For a proof of this proposition see $[14$, Section 4.6]. In the real analytic case we even obtain a generic set in $\mathcal{U}$ independent of $x \in M$, i.e. the set $\{u \in \mathcal{U}$, for all $x \in M$ there exists $T>0$ such that $\varphi(t, x, u) \in C\}$ for all $t \geq T$ is open and dense in $\mathcal{U}$. In this case, a stochastic system satisfies the corresponding property w.p.1., if the $\theta$-invariant measure $P$ on $\mathcal{U}$ puts probability one on the generic set, which, of course, is in general difficult to verify. However, some general statements can be made about the limit behavior of perturbed stochastic systems, which we summarize below. For this we need some facts on invariant measures and recurrence.

Definition 3.23. A point $x \in S$ is called recurrent for a flow $\Psi$ on $S$ if $x \in \omega(x)$. A probability measure $\mu$ on the Borel $\sigma$-algebra of $S$ is called invariant for $\Psi$ if $\Psi_{t} \mu=\mu$ for all $t \in \mathbb{R}$, and ergodic if $\mu\left(A \Delta \Psi_{-t} A\right)=0$ for all $t \in \mathbb{R}$ implies $\mu A=0$ or $\mu A=1$. Here $\Delta$ denotes the symmetric difference of two sets.

Poincaré's Recurrence Theorem states that on a separable metric space $S$ we have $\mu\{x \in S, x \notin \omega(x)\}=0$ for any $\Psi$-invariant measure $\mu$, in other words, $\mu$-almost all points are recurrent (compare, e.g. [33, Prop. I.2.1]). Hence the support of any $\psi$-invariant measure is contained in the closure of the set $\mathcal{R}^{\#}$ of $\psi$-recurrent points. However, the union of the supports of all invariant measures need not be dense in cl $\mathcal{R}^{\#}$, compare, e.g. [34, Sec. VI.3].

For the flows (5) of perturbed systems the situation is simpler, as the following results show. Here we denote by $\mathcal{P}_{\Phi}$ the set of $\Phi$-invariant measures on $\mathcal{U} \times M$. This set is nonempty, convex, weakly compact, and the extremal points are ergodic measures, compare [27, Lemma 4.1.10].

Proposition 3.24. Consider the system flow (5) under the Lie algebra rank condition (14).

(i) For every $(u, x) \in \mathcal{U} \times M$ there exists a chain control set $E$ of (4) such that $\omega(u, x) \subset \mathcal{E}$, the lift of $E$, and hence $\pi_{M} \omega(u, x) \subset E$.

(ii) For all $(u, x) \in \mathcal{U} \times M$ we have supp $\mu_{u, x} \subset \omega(u, x)$, where $\mu_{u, x}$ is the Krylov-Bogolyubov invariant measure from $(u, x)$, compare, e.g. [34, Th. VI.9.05].

(iii) If $(u, x) \in \mathcal{U} \times M$ is $\Phi$-recurrent, then there exists a control set $D$ of (4) with $x \in D$.

(iv) If $D$ is a main control set of (4), then for every $x \in D$ there exists $u \in \mathcal{U}$ such that $(u, x)$ is $\Phi$-recurrent.

These results follow from [14, Cor. 4.3.12, Prop. 4.4.1, 4.4.2, Th. 4.4.6]. As a consequence we obtain a characterization of the possible supports of all $\Phi$-invariant measures. 
Theorem 3.25. Consider the system flow (5) under the Lie algebra rank condition (14). For a control set $D$ of (4) denote the positive lift by $\mathcal{D}^{+}=$ $\operatorname{cl}\{(u, x) \in \mathcal{U} \times M, \varphi(t, x, u) \in D$ for all $t \geq 0\}$ and set $\mathcal{D}^{\#}=\bigcup\left\{\mathcal{D}^{+}, D\right.$ is control set\}.

(i) $\bigcup\left\{\right.$ supp $\left.\mu, \mu \in \mathcal{P}_{\Phi}\right\} \subset \mathrm{cl} \mathcal{D}^{\#}$.

(ii) If all control sets of (4) are contained in the closure of main control sets, then $\mathrm{cl} \bigcup\{D, D$ is main control set $\}=\pi_{M} \mathrm{cl} \bigcup\{$ supp $\mu, \mu \in$ $\left.\mathcal{P}_{\Phi}\right\}=\pi_{M} \mathcal{R}^{\#}$, the projection of all $\Phi$-recurrent points.

(iii) For each $\Phi$-invariant ergodic measure $\mu$ there exists one control set $D$ of (4) such that supp $\mu \subset \mathcal{D}^{+}$.

This result (compare [14, Cor. 4.4.7, 4.4.8]) concerns the existence and location (supports) of $\Phi$-invariant measures as well as their relation to recurrent points. In particular, all projections of recurrent points and of control sets are contained in the closures of control sets. In general, a system flow (5) will have many invariant measures, even over the same control set.

Consider now the stochastic perturbation model (5) with a given $\theta-$ invariant probability measure $P$ on $\mathcal{U}$. It is easy to see that a measure $\mu$ on $\mathcal{U} \times M$ is $\Phi$-invariant iff $\mu(d u, d x)=\mu_{u}(d x) P(d u)$, where $P$ is $\theta$-invariant and $\mu_{u}=\Phi(t, u, \cdot) \mu_{\theta(t, u)}$. Measures of this type are studied, e.g. in [3] and [15]. The existence of an invariant family $\left\{\mu_{u}, u \in \mathcal{U}\right\}$ is always guaranteed over invariant control sets of (4) (recall that we assume $M$ to be compact, hence any invariant control set $C$ is compact and $\mathcal{U} \times C$ is $\Phi$-invariant for $t \geq 0$ ). The existence of invariant families over noninvariant control sets $D$ boils down to the question whether $\Phi(t, u, \cdot)$ leads out of $D$ with positive $P$-probability. We discuss one set of conditions under which this is true. The condition is modelled after the support theorem, which was the crucial tool in the Markov diffusion case above (but for Markov invariant measures).

Let $\{\eta(t), t \in \mathbb{R}\}$ be a stationary stochastic process with trajectory space $\mathcal{U}$ and $\theta$-invariant measure $P$. Denote by supp $P_{\eta(0)}$ the support of the distribution of $\eta(0)$ in $U$. We use the following assumption (compare [5, p. 16]).

There exists $y_{0} \in \operatorname{supp} P_{\eta(0)}$ such that for all $\delta>0$ and all $u:[0, T] \rightarrow U$ continuous with $u(0)=y_{0}$

we have $P\left\{\max _{0 \leq t \leq T}|\eta(t)-u(t)|<\delta\right\}>0$.

Theorem 3.26. Consider the system flow (5) with stationary stochastic perturbation satisfying (23). Assume the Lie algebra rank condition (14).

(i) For any point $x \in D$, some control set of (4), we have for all $\epsilon>0$ and all $T>0$ that $P\{d(\varphi(t, x, \omega), x)<\varepsilon$ for some $t \geq T\}>0$. 
(ii) Any $\Phi$-invariant measure $\mu$ with marginal $P$ on $\mathcal{U}$ satisfies $\pi_{M}$ supp $\mu$ $\cap C \neq \emptyset$, where $C=\bigcup_{i=1}^{k} C_{i}$ is the union of the invariant control sets of (4).

Proof. Part (i) was proved in [5, Prop. 3.6]. Part (ii) follows from Proposition 3.22 (i) and the 'real noise tube method' in [5, p. 18].

Under further conditions on the control structure of (4) one can obtain stronger results.

Corollary 3.27. Under the conditions of Theorem 3.26 assume that all invariant control sets $C_{i}, i=1 \ldots k$ of (4) are isolated, i.e. for all $i$ there exists $\alpha>0$ such that the open $\alpha$-neighborhood $B\left(C_{i}, \alpha\right)$ does not intersect any other control set. Then any ergodic invariant measure $\mu$ satisfies $\pi_{M}$ supp $\mu \subset C_{i}$ for some $i=1 \ldots k$.

Note that by Kunita's theorem (13) the Markov diffusion model (9) satisfies the condition (23). In fact, this model satisfies (23) for all $y_{0} \in U$. Hence we obtain

Corollary 3.28. Consider the system flow (5) for the Markov diffusion case (9) under the Lie algebra rank conditions (7) and (20). Then any $\Phi-$ invariant measure $\mu$ with marginal $P$ on $\mathcal{U}$ satisfies $\pi_{M}$ supp $\mu \subset C$, where again $C$ is the union of the finitely many invariant control sets $C_{i}, i=$ $1 \ldots k$ of (4). Furthermore, each ergodic $\Phi$-invariant measure has support, whose projection lies in one of the $C_{i}$.

The result forces us to study the relation between the invariant Markov measures (22) and the invariant measures of the system flow. Each invariant Markov measure induces a $\Phi$-invariant measure, but, in general, the system flow may have many more invariant measures, compare [15] for a thorough discussion of this topic. Even if a $\Phi$-invariant measure $\mu$ is induced by a Markov measure, the study of its family $\left\{\mu_{u}, u \in \mathcal{U}\right\}$ reveals additional detail information that cannot be seen from the state space density of the Markov measure. These families are studied in detail in [3] and results for specific systems are presented, e.g. in [17], [37], and [4], where the last two articles contain mainly numerical results.

\subsection{Attractors}

We conclude this section with a brief discussion of attractors in perturbed systems. The starting point are again some results for continuous flows $\Psi: \mathbb{R} \times S \rightarrow S$ on compact metric spaces.

Definition 3.29. A compact invariant set $A \subset S$ is an attractor of $(S, \Psi)$ if it admits a neighborhood $N$ such that $\omega(N)=A$. A repeller is a compact invariant set $R \subset S$ which has a neighborhood $N^{*}$ with $\omega^{*}\left(N^{*}\right)=R$. For 
an attractor $A$ the set $A^{*}=\{x \in S, \omega(x) \cap A=\emptyset\}$ is a repeller, called the complementary repeller, the pair $\left(A, A^{*}\right)$ is called an attractor-repeller pair.

The relation between attractors and Morse decompositions, see Definition 3.4 , is described in the next result.

Lemma 3.30. A finite collection $\left\{\mathcal{M}_{1}, \ldots, \mathcal{M}_{n}\right\}$ of subsets of $S$ is a Morse decomposition iff there is a strictly increasing sequence of attractors

$\phi=A_{0} \subset A_{1} \subset \ldots \subset A_{n}=S$ such that $\mathcal{M}_{n-i}=A_{i+1} \cap A_{i}^{*}$ for $0 \leq i \leq n-1$.

Hence the attractor-repeller pairs can be reconstructed once all Morse decompositions are known. The relation between attractors and the chain recurrent set, compare Definition 3.3, is as follows (see [14, Prop. B.2.24 and Th. B.2.25]).

Lemma 3.31. (i) For $V \subset S$ the chain orbit $\Omega(V)$ is the intersection of all attractors containing $\omega(V)$.

(ii) The chain recurrent set $\mathcal{R}$ satisfies $\mathcal{R}=\cap\left\{A \cup A^{*}, A\right.$ is an attractor $\}$. Hence if the flow $(S, \Psi)$ has a finest Morse decomposition $\left\{\mathcal{M}_{1}, \ldots, \mathcal{M}_{n}\right\}$, then the Morse sets are the components of $\mathcal{R}$ and each such component is the intersection of unions of attractor-repeller pairs.

Note that the flow $(S, \Psi)$ has at most countably many attractors (see [36, Lemma 9.1.7].

We apply these results to the flow (5) of perturbed dynamical systems. Note first of all that the perturbation model (3) has only the trivial attractors $\emptyset$ and $\mathcal{U}$. Hence the attractors of the system flow (5) result from the skew component $\varphi$. For systems with finitely many chain recurrent components the chain transitive attractors are the most important ones since, by the results above, they form the nuclei of attractor sequences. For the flows of perturbed systems these attractors are given by certain chain control sets, compare [14, Prop. 4.3.19].

Theorem 3.32. Consider the system flow (5) and the associated control system (4).

(i) Let $\left(A, A^{*}\right)$ be an attractor-repeller decomposition of $(\mathcal{U} \times M, \Phi)$ such that $A$ is a chain recurrent component. Then there exists a chain control set $E$, maximal w.r.t. the order $\preceq$ defined in Corollary 3.16, such that

$$
A=\mathcal{E} \text { and } A^{*}=\mathcal{A}(\mathcal{E})^{c},
$$

where $\mathcal{E}$ is the lift of $E$ from (18), and $\mathcal{A}(\mathcal{E})=\{(u, x) \in \mathcal{U} \times M, \omega(u, x) \subset$ $\varepsilon\}$.

(ii) If the number of chain control sets of (4) is finite, then for every maximal (w.r.t. $\preceq$ ) chain control set $E$ there is an attractor-repeller pair $\left(A, A^{*}\right)$ such that (24) holds for the lift $\mathcal{E}$ of $E$. 
Note that without the finiteness assumption part (ii) of the theorem is false. According to this theorem, maximal chain control sets in $M$ and chain recurrent attractors in $\mathcal{U} \times M$ coincide (under the finiteness assumption), and hence these 'minimal' attractors (which do not contain other attractors except for the empty set) can be characterized in the state space $M$ of the perturbed system. This is, in general, not true for other attractors, as the existence of multistable points shows. Under the Lie algebra rank condition (14), Proposition 3.22 shows that for attractors $\emptyset \neq A_{1} \subset A_{2} \subset$ $\mathcal{U} \times M, A_{1} \neq A_{2}$ the set $A_{2} \backslash A_{1}$ is topologically thin, i.e. contained in the complement of an open and dense set.

For stochastically perturbed systems the appropriate concept of a 'stochastic attractor' is not so obvious. Recall that the $\omega$-limit set of $V \subset \mathcal{U} \times M$ is defined via sequences in time and in $\mathcal{U} \times M$, with the consequence that $c l(\cup\{\omega(x, u),(u, x) \in V\}) \subset \omega(V)$. Hence it is not obvious, how to combine the given $\theta$-invariant measure $P$ on $\mathcal{U}$ with sequences $\left(u_{n}, x_{n}\right)$ in $V$. One possibility is to think of a 'stochastic attractor' as a set in the state space $M$ that contains all $\omega$-limit sets of random trajectories starting in some neighborhood if this set. We refer the reader to [16] and [7] for concepts and results along these lines. In any case, if the stochastic perturbation process is sufficiently nondegenerate, a result analogous to Theorem 3.32 should hold. These and other problems concerning the characterization of 'stochastic attractors' seem to be open at this moment.

Remark 3.33. (On numerical methods). The results presented in this section require the numerical computation of main and chain control sets, their lifts, and of limit sets if the theory is to be applied to concrete examples. For the computation of main control sets several algorithms are available: based on the numerical solution of families of ordinary differential equations [23], based on the solution of time optimal control problems (see 114, Appendix C.3]), and based on subdivision techniques [407, which were developed for dynamical systems by Dellnitz and Hohmann. Since chain control sets are 'almost always' the closures of main control sets (compare Theorem 4.2 below) these algorithms also serve for chain control sets. The computation of objects in $\mathcal{U} \times M$ and their $u$-wise projection onto $M$ requires algorithms for time-varying differential equations, whose limit sets are, in general, fairly complex. We refer to [6] and to [4] in this volume for a discussion of several approaches in the stochastic context.

In this section we saw that the global behavior of Markov diffusion systems is determined by the orbit structure, i.e. the trajectories of the associated control system. In contrast, the limit sets, supports of invariant measures, and attractors of general perturbed flows follow the topological chain structure of these flows. In general, these two structures can be different. However, in the next section we show that under an inner pair condition the structures agree 'almost always'. Hence the study of Markov diffusion systems can be related to the topology of the corresponding per- 
turbed flow (2).

\section{Global Behavior of Parameter Dependent Perturbed Systems}

This section serves mainly two purposes: to clarify the relation between the control and the chain control structure of the system flow (5), and to study the global picture that was developed in Section 3 under the variation of system parameters. We concentrate on the topological properties of the flow (5), the consequences for stochastically perturbed systems follow from a direct application to the stochastic results in Section 3. None of the results in this section is new, the proofs can be found in [14], Chapters 3. and 4., primarily in Section 4.7. The model of perturbed systems, as introduced in Section 3, can basically depend on parameters in two ways: The vector fields in the system equation (4) may depend on a parameter $\alpha \in I \subset \mathbb{R}^{p}$, and the perturbation range $U \subset \mathbb{R}^{m}$ may vary. Therefore, we consider the following family of perturbed systems on the compact $C^{\infty}$-manifold $M$, parametrized by $(\alpha, \rho) \in I \times[0, \infty)$

$$
\begin{aligned}
& \dot{x}=X_{0}(x, \alpha)+\sum_{i=1}^{m} u_{i}(t) X_{i}(x, \alpha)=X(x, u, \alpha), \alpha \in I, \\
& u \in \mathcal{U}^{\rho}=\left\{u: \mathbb{R} \rightarrow U^{\rho}, \text { measurable }\right\} \\
& U^{\rho}=\rho \cdot U \text { for } \rho \geq 0, U \subset \mathbb{R}^{m} \text { convex and compact with } 0 \in \operatorname{int} U .
\end{aligned}
$$

which leads to a continuous system flow

$$
\Phi^{\alpha, \rho}: \mathbb{R} \times \mathcal{U}^{\rho} \times M \rightarrow \mathcal{U}^{\rho} \times M, \Phi_{t}(u, x)=\left(\theta_{t} u, \varphi(t, x, u)\right) .
$$

In the case of a stochastic perturbation model the shift invariant measure $P$ may also depend on a parameter, e.g., if the vector fields of the background noise (6) on $N$ are parameter dependent. We do not consider this situation in the current paper, except for the fact that we vary the noise range, i.e. for the Markov diffusion model we consider a family of maps $f: N \rightarrow U^{\rho}$ as in (8) and Lemma 3.17.

We continue to deal with regular systems and assume a Lie algebra rank condition (compare (14)) of the form

$\operatorname{dim} \mathcal{L} \mathcal{A}\left\{X_{0}(\alpha)+\sum u_{i} X_{i}, u \in U^{\rho}\right\}(x)=\operatorname{dim} M$ for all $x \in M, \alpha \in I, \rho>0$.

The following results require a relation between the limit sets of the system flow $\Phi^{\alpha, \rho}$ and the control sets of $(25)^{\alpha, \rho}$. For this we impose a so-called 
inner pair condition:

Fix $\alpha \in I$. For all $\rho, \rho^{\prime} \in\left[0, \rho^{*}\right)$ with $\rho<\rho^{\prime}$ and all chain control sets $E^{\rho}$ of $(25)^{\alpha, \rho}$ every $(u, x) \in \mathcal{E}^{\rho} \subset \mathcal{U}^{\rho} \times M$ satisfies:

There exists $T(u, x)>0$ such that $\varphi(T, x, u) \in \operatorname{int} \mathcal{O}^{+, \rho^{\prime}}(x)$, the positive orbit of $x \in M$ under the control range $U^{\rho^{\prime}}$.

This condition says that the trajectories in chain control sets of $(25)^{\rho}$ enter the interior of the corresponding orbits of $(25)^{\rho^{\prime}}$ for $\rho^{\prime}>\rho$. In fact, Condition (28) is too strong for many of the results below, and we refer the reader to $[14$, Chapter 4] for weaker versions and for sufficient conditions which imply (28).

Our first result shows that, depending on $(\alpha, \rho)$, control sets and chain control sets enjoy complementary semicontinuity properties in the Hausdorff topology on subsets of $M$.

Theorem 4.1. Consider the system flow (26) ${ }^{\alpha, \rho}$ under Assumptions (27) and (28). Assume that the vector fields $X_{0}, \ldots, X_{m}$ in (26) ${ }^{\alpha, \rho}$ have $C^{\infty}$ dependence on $\alpha$.

(i) For $\left(\alpha_{0}, \rho_{0}\right) \in$ int $I \times(0, \infty)$ let $D^{\alpha_{0}, \rho_{0}}$ be a main control set of $(25)^{\alpha, \rho}$. Then there are unique control sets $D^{\alpha, \rho}$ such that the map $(\alpha, \rho) \mapsto$ cl $D^{\alpha, \rho}$ on $I \times(0, \infty)$ is lower semicontinuous at $\left(\alpha_{0}, \rho_{0}\right)$.

(ii) Pick $\left(\alpha_{0}, \rho_{0}\right) \in I \times[0, \infty)$ and consider for a sequence $\left(\alpha_{k}, \rho_{k}\right) \rightarrow$ $\left(\alpha_{0}, \rho_{0}\right)$ chain control sets $E^{\alpha_{k}, \rho_{k}}$. Then there exists a chain control set $E^{\alpha_{0}, \rho_{0}}$ such that

$$
\begin{aligned}
& \quad \limsup _{\left(\alpha_{k}, \rho_{k}\right) \rightarrow\left(\alpha_{0}, \rho_{0}\right)} E^{\alpha_{k}, \rho_{k}}:= \\
& \quad\left\{x \in M, \text { there are } x^{k} \in E^{\alpha_{k}, \rho_{k}} \text { with } x_{k} \rightarrow x\right\} \subset E^{\alpha_{0}, \rho_{0}},
\end{aligned}
$$

i.e. the $\operatorname{map}(\alpha, \rho) \mapsto E^{\alpha, \rho}$ on $I \times[0, \infty)$ is upper semicontinuous.

In general, one cannot expect the maps in Theorem 4.1 to be continuous, even if $\alpha$ or $\rho$ are fixed. However, for $\alpha$ fixed these maps are continuous almost everywhere in $\rho$.

Fix $\alpha \in I$ and consider the interval $\left[\rho_{*}, \rho^{*}\right] \subset[0, \infty)$. Let $E^{\rho_{*}}$ be a chain control set of $(25)^{\rho}$ and define the maps into the compact subsets $\mathcal{K}(M)$ of $M$

$$
\begin{aligned}
{\left[\rho_{*}, \rho^{*}\right) \rightarrow \mathcal{K}(M), \rho } & \mapsto E^{\rho} \text { with } E^{\rho_{*}} \subset E^{\rho} \\
\left(\rho_{*}, \rho^{*}\right] \rightarrow \mathcal{K}(M), \rho & \mapsto \operatorname{cl} D^{\rho} \text { with } E^{\rho_{*}} \subset D^{\rho}
\end{aligned}
$$

Theorem 4.2. Consider the system flow (26) for fixed $\alpha \in I$ under Assumptions (27) and (28).

(i) The map (29) is well defined, increasing, and right continuous. 
(ii) The map (30) is well defined, increasing, and left continuous.

(iii) For $\rho_{*} \leq \rho<\rho^{\prime} \leq \rho^{*}$ we have $D^{\rho} \subset E^{\rho} \subset$ int $D^{\rho^{\prime}}$.

(iv) The continuity points of (29) and (30) agree and at each continuity point $\rho$ we have cl $D^{\rho}=E^{\rho}$. Furthermore, there are at most countably many points of discontinuity.

According to this theorem, under the inner pair condition (28) the chain control sets are 'almost always' closures of main control sets. This clarifies the connection between the chain structure and the orbit structure of perturbed systems that was used in Section 3 for the study of Markov diffusion systems. To complete the analysis of the relation between the control structure and the chain control structure of control systems, we study the different orders that were defined in (16) and Corollary 3.16.

Theorem 4.3. Consider the system flow (26) for fixed $\alpha \in I$ under (27) and (28), and assume that (26) ${ }^{\rho_{*}}$ has finitely many chain control sets $E_{1}^{\rho_{*}}, \ldots, E_{k}^{\rho_{*}}$.

(i) If $E_{i}^{\rho_{*}} \preceq E_{j}^{\rho_{*}}$, then $D_{i}^{\rho} \preceq D_{j}^{\rho}$ for all $\rho \in\left(\rho, \rho^{*}\right]$.

(ii) If there are $\rho_{n} \downarrow \rho_{*}$ with $D_{i}^{\rho_{n}} \preceq D_{j}^{\rho_{n}}$ for all $n \in \mathbb{N}$, then $E_{i}^{\rho_{*}} \preceq E_{j}^{\rho_{*}}$.

(iii) For $\rho>\rho_{*}, \rho-\rho_{*}$ small enough the invariant control sets of $(25)^{\rho}$ correspond uniquely to the maximal (w.r.t. $\preceq$ ) chain control sets of $(25)^{\rho_{*}}$.

These results open the door for a global analysis of parameter dependent perturbation systems. For the moment, fix $\alpha \in I$ and consider the system $(25)^{\alpha, \rho},(26)^{\alpha, \rho}$ with varying perturbation range $U^{\rho}, \rho \geq 0$. We start from the unperturbed system (i.e. $\rho=0$ )

$$
\dot{x}=X_{0}(x, \alpha) \text { on } M, \quad \Phi^{0}: \mathbb{R} \times M \rightarrow M,
$$

and assume that $\left(31^{\alpha}\right)$ has a finest Morse decomposition $\left\{\mathcal{M}_{1}, \ldots, \mathcal{M}_{n}\right\}$. As $\rho$ increases from 0 , chain control sets (and hence main control sets) form around the $\mathcal{M}_{i}$ (note that the map (29) is continuous at $\rho=0$ ), preserving the order given by the Morse decomposition. In particular, invariant control sets form around the maximal Morse sets. However, it is not true, in general, that the number of (main) control sets, even for $\rho>0$ small, agrees with the number of Morse sets of $\left(31^{\alpha}\right)$, compare [14, Example 4.7.8]. At a discontinuity point of the maps (29) and (30) the size of the (chain) control sets jumps, often through the merging of different control sets. Note that by the existence of multistability regions $M S$ (compare Definition 3.18) two invariant control sets can only merge for $\rho>0$ if their union also includes a set that was a noninvariant control set for $\rho^{\prime}<\rho$. Concrete results for specific systems can be found, e.g., in [14, Chapters 8,9 , and 13]. For the 
Markov diffusion model the situation seems to be relatively simple, because its invariant measures and stationary solutions live exactly on the invariant control sets, compare Theorem 3.20 and Corollary 3.28. In fact, for systems satisfying Assumptions (27) and (28) the parameter dependence of the support of the invariant Markov measures is described through the results above, compare [11], [9], [13] for several concrete examples. However, a study of the families $\left\{\mu_{u}, u \in \mathcal{U}^{\rho}\right\}$ of the induced flow invariant measures, compare Corollary 3.28 and the discussion thereafter, is still missing (compare, however, [18] for the one-dimensional white noise case). This is true, even more so, for the general stochastic perturbed model.

Finally, let us consider the parametric system flow $(26)^{\alpha, \rho}$ depending on $\alpha$ and $\rho$. For a given bifurcation scenario (in $\alpha \in I$ ) of the Morse sets of the unperturbed system $\left(31^{\alpha}\right)$, Theorems 4.1 to 4.3 show that this scenario can be recovered by a two parameter approximation in $\alpha$ and $\rho \downarrow 0$ through perturbation systems of the type (26) ${ }^{\alpha, \rho}$, compare [12] for the one-dimensional and [14, Sec. 9.2] for the Hopf bifurcation case. Along the same lines one studies the continuity of the support of invariant Markov measures. The field for a further analysis of stochastic perturbation models is wide open.

Acknowledgement The authors acknowledge the help of the Bremen group in preparing this manuscript, and we thank the referees for their constructive criticism.

\section{REFERENCES}

[1] E. Akin. The General Topology of Dynamical Systems. Amer. Math. Soc., Providence, R.I., 1993.

[2] L. Arnold. Stochastic Differential Equations: Theory and Applications. Wiley, 1974. Original German language edition 1973 by Oldenbourg Verlag, München; English edition reprinted by Krieger, Malaba (Florida), 1992.

[3] L. Arnold. Random Dynamical Systems. Springer-Verlag, Berlin Heidelberg New York, 1998.

[4] L. Arnold, G. Bleckert, and K. R. Schenk-Hoppé. The stochastic Brusselator: Parametric noise destroys Hopf bifurcation. In H. Crauel and V. M. Gundlach, editors, Stochastic Dynamics. Springer-Verlag, 1998.

[5] L. Arnold and W. Kliemann. Qualitative theory of linear stochastic systems. In A. Bharucha-Reid, editor, Probabilistic Analysis and Related Topics, Vol 3, pages 1-79. Academic Press, 1983. 
[6] L. Arnold, N. S. Namachchivaya, and K. R. Schenk-Hoppé. Toward an understanding of stochastic Hopf bifurcation. Intern. J. Bifur. Chaos Appl. Sci. Engrg., 6:1947-1975, 1996.

[7] L. Arnold and B. Schmalfuß. Fixed points and attractors for random dynamical systems. In A. Naess and S. Krenk, editors, IUTAM Symposium on Advances in Nonlinear Stochastic Mechanics, pages $19-$ 28. Kluwer, 1996.

[8] P. H. Baxendale. Brownian motion in the diffeomorphism group I. Compositio Math., 53:19-50, 1984.

[9] F. Colonius, F. J. de la Rubia, and W. Kliemann. Stochastic models with multistability and extinction levels. SIAM J. Appl. Math., 56:919-945, 1996.

[10] F. Colonius and W. Kliemann. Some aspects of control systems as dynamical systems. J. Dynamics and Differential Equations, 5:469494, 1993.

[11] F. Colonius and W. Kliemann. Random perturbations of bifurcation diagrams. Nonlinear Dynamics, 5:353-373, 1994.

[12] F. Colonius and W. Kliemann. Controllability and stabilization of one dimensional control systems near bifurcation points. Syst. Control Letters, 24:87-95, 1995.

[13] F. Colonius and W. Kliemann. Nonlinear systems with multiplicative and additive perturbation under state space constraints. Active/Passive Vibration Control and Nonlinear Dynamics of Structures, W. W. Clark et al., eds., Amer. Soc. Mech. Eng. ASME-DE, 84-1:365$373,1997$.

[14] F. Colonius and W. Kliemann. The Dynamics of Control. Birkhäuser, 1998. to appear.

[15] H. Crauel. Markov measures for random dynamical systems. Stochastics and Stochastics Reports, 37:153-173, 1991.

[16] H. Crauel and F. Flandoli. Attractors for random dynamical systems. Probab. Theory Relat. Fields, 100:365-393, 1994.

[17] H. Crauel and F. Flandoli. Additive noise destroys a pitchfork bifurcation. J. Dynamics Diff. Equations, 10:259-274, 1998.

[18] H. Crauel, P. Imkeller, and M. Steinkamp. Bifurcation of onedimensional stochastic differential equations. In H. Crauel and V. M. Gundlach, editors, Stochastic Dynamics. Springer-Verlag, 1998. 
[19] R. Devaney. An Introduction to Chaotic Dynamical Systems. Benjamin/Cummings, 1986.

[20] E. Dynkin. Markov Processes I. Springer-Verlag, Berlin, 1965.

[21] S. Ethier and T. Kurtz. Markov Processes. Wiley, 1986.

[22] I. Gichman and A. Skorochod. The Theory of Stochastic Processes I, II, III. Springer-Verlag, New York, 1973, 1974, 1975.

[23] G. Häckl. Reachable sets, control sets and their computation. Dissertation, Universität Augsburg, "Augsburger Mathematische Schriften Band 7", 1996.

[24] K. Ichihara and H. Kunita. A classification of the second order degenerate elliptic operators and its probabilistic characterization. $Z$. Wahrsch. verw. Gebiete, 30/39:235-254/81-84, 1977.

[25] N. Ikeda and S. Watanabe. Stochastic Differential Equations and Diffusions Processes. North Holland, 1981.

[26] V. Jurdjevic. Geometric Control Theory. Cambridge University Press, 1997.

[27] A. Katok and B. Hasselblatt. Introduction to the Modern Theory of Dynamical Systems. Cambridge University Press, 1995.

[28] R. Z. Khasminskii. Stochastic Stability of Differential Equations. Sijthoff and Noordhoff, Alphen an der Rijn, 1980. Russian original: Nauka, Moscow, 1969.

[29] W. Kliemann. Recurrence and invariant measures for degenerate diffusions. Ann. Prob., 15:690-707, 1987.

[30] H. Kunita. Diffusion Processes and Control Systems. University of Paris, 1974. Lecture notes.

[31] H. Kunita. Supports of diffusion processes. In K. Ito, editor, Proceedings Int. Symp. Stochastic Diff. Equ., pages 163-185. Wiley, 1978.

[32] H. Kunita. Stochastic Flows and Stochastic Differential Equations. Cambridge University Press, 1990.

[33] R. Mané. Ergodic Theory and Differentiable Dynamics. SpringerVerlag, 1987.

[34] V. Nemytskii and V. Stepanov. Qualitative Theory of Dynamical Systems. Princeton University Press, 1960. Russian original 1949. 
[35] V. Oseledets. A multiplicative ergodic theorem. Lyapunov characteristic numbers for dynamical systems. Trans. Moscow Math. Soc, 19:197-231, 1968.

[36] C. Robinson. Dynamical Systems. Stability, Symbolic Dynamics, and Chaos. CRC Press Inc., 1995.

[37] K. R. Schenk-Hoppé. Bifurcation scenarios for the noisy Duffing-van der Pol oscillator. Nonlinear Dynamics, 11:255-274, 1996.

[38] G. Sell. Lectures on Linear Differential Systems. School of Mathematics, University of Minnesota, Minneapolis, Minnesota, 1975.

[39] D. Stroock and S. Varadhan. On the support of diffusion processes with applications to the strong maximum principle. In Proc. 6th Berkeley Symp. Math. Stat. Probab. Vol 3, pages 333-359, 1972.

[40] D. Szolnoki. Berechnung von Viabilitätskernen. Diplomarbeit, Institut für Mathematik, Universität Augsburg, Augsburg, 1997. 